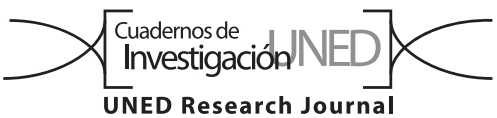

\title{
Contaminación y disminución del embalse El Laguito, Costa Rica, por aguas urbanas: encuesta y propiedades físico-químicas
}

\author{
María José Chassoul Acosta' ${ }^{1}$ \& Cindy Rodríguez-Arias ${ }^{2}$ \\ 1. Ingeniería Industrial, Sede de Occidente de la Universidad de Costa Rica. San Ramón, Alajuela, Costa Rica. Apdo. 111-4250; \\ maria.chassoul@ucr.ac.cr \\ 2. Sección de Biología, Sede de Occidente de la Universidad de Costa Rica. San Ramón, Alajuela, Costa Rica. Apdo. 111-4250; \\ cindy.rodriguez_a@ucr.ac.cr
}

Recibido 10-II-2018 • Corregido 09-V-2018 • Aceptado 23-VI-2018

\begin{abstract}
Pollution and reduction of the El Laguito reservoir, Costa Rica, by urban waters: survey and physical-chemical properties. The pollution and size reduction of water reservoirs have an important impact on tropical biodiversity. The Laguito, Sede de Occidente, in Alajuela, Costa Rica, is an artificial reservoir built for recreational purposes, but currently works as a rainwater reservoir to prevent floods that occur in the lower areas of the microbasin. In recent years, it has been reduced in size and lost the water mirror and the reservoir capacity is very small. To assess water management by the neighbors, we surveyed 92 nearby homes that discharge waste water to El Laguito through a sewer and a pipe. We also took water samples in April and September of 2015 and July of 2016 for standard physic-chemical analysis. The neighbors waste water and $80 \%$ of the houses discharge the untreated raw waters into the system. An educational program would improve practices in the area and benefit the reservoir and the associated organisms.
\end{abstract}

Key words: Water management, waste water, contamination, El Laguito, hydric resource.
RESUMEN: La contaminación y la reducción del tamaño de los reservorios de agua tienen un impacto importante en la biodiversidad tropical. El Laguito de la Sede de Occidente, en Alajuela, Costa Rica, es un embalse artificial construido con fines recreativos, pero funciona actualmente como reservorio de agua de lluvia para prevenir las inundaciones que se presentan en las zonas bajas de la microcuenca. En los últimos años, se ha reducido su tamaño y ha perdido el espejo de agua y la capacidad del depósito es muy pequeña. Para evaluar el manejo del agua por parte de los vecinos, encuestamos los 92 hogares cercanos que descargan aguas residuales a El Laguito a través de un alcantarillado y una tubería. También tomamos muestras de agua en abril y setiembre de 2015 y julio de 2016 para el análisis fisicoquímico estándar. Los vecinos desperdician agua y el $80 \%$ de las casas descargan las aguas crudas $\sin$ tratar en el sistema. Un programa educativo mejoraría las prácticas en el área y beneficiaría al yacimiento y los organismos asociados.

Palabras claves: Manejo de agua, aguas residuales, contaminación, El Laguito, recurso hídrico.
El aumento poblacional, el crecimiento urbanístico e industrial, así como la intensificación de las actividades agrícolas y pecuarias han generado un aumento en la frecuencia y cantidad de residuos que se descargan a los cuerpos de agua superficiales, donde la mayoría están afectados en su calidad por aportes puntuales y no puntuales de materiales que van desde sedimentos, aguas negras, residuos industriales y agropecuarios, agroquímicos y residuos sólidos. La información disponible indica que en Costa Rica más del $70 \%$ de las aguas negras sin tratamiento llegan a nuestros ríos (MINAET, SENARA \& AyA, 2012).

El ciclo natural del agua tiene una gran capacidad de purificación, pero esta misma facilidad de regeneración y la aparente abundancia han hecho que sea el vertedero habitual de residuos. En conjunto con esto, las inadecuadas prácticas de gestión han ocasionado que en la actualidad nos enfrentemos a una importante crisis en el ámbito de los recursos hídricos desde el punto de vista cuantitativo y cualitativo (Estrada, López, Vásquez, Sánchez \& Ruvalcaba, 2016).

Los altos niveles de contaminación alcanzados hacen que el agua no sea apta para consumo humano, por lo que es necesario sistemas de tratamiento cada vez más complejos para poder purificarla (Chulluncuy, 2011). Muchas ciudades del mundo carecen de buena calidad de agua y los habitantes enferman debido a la presencia 
de microorganismos patógenos, sustancias tóxicas o nocivas para la salud en ésta.

Un caso que hace referencia a lo anterior se da en la provincia de Alajuela, Costa Rica, en donde se construyó un embalse artificial con aguas de la quebrada El Estero, con el fin de funcionar, inicialmente, como reservorio de agua para fines recreativos. Pero luego ha funcionado como reservorio de agua de lluvia para prevenir las inundaciones que se presentan en las zonas bajas de la microcuenca. Sin embargo, dada la contaminación de la quebrada, la falta de un adecuado manejo del sitio, la acumulación de sedimentos y posterior invasión de plantas, se ha perdido por completo el espejo de agua y la capacidad de embalse se ha reducido (Mora, 2007).

Un estudio realizado por Rodríguez y Silva (2015), determinó que la descarga de aguas residuales domésticas es la principal causa del deterioro de la calidad del agua de la quebrada y del embalse. Por ello, el objetivo de esta investigación es evaluar el manejo de las aguas que ingresan directamente al embalse El Laguito provenientes de las urbanizaciones, con el fin de plantear una alternativa de solución que permita disminuir el nivel de contaminación y, a la vez, que los resultados obtenidos sirvan como insumo para el posterior planteamiento de una estrategia de gestión que permita solucionar el problema.

\section{METODOLOGÍA}

El embalse El Laguito se encuentra dentro de la Sede de Occidente de la Universidad de Costa Rica (10'05'47"$10^{\circ} 04^{\prime} 31^{\prime \prime} \mathrm{N}$ y $84^{\circ} 28^{\prime} 20^{\prime \prime}$ y $\left.84^{\circ} 29^{\prime} 41^{\prime \prime} \mathrm{O}\right)$, en la provincia de Alajuela. Se ubica dentro de la microcuenca Estero, que a su vez está dentro de la subcuenca del río Grande de San Ramón y de la cuenca del río Grande de Tárcoles (Fig. 1). El área aproximada es de $1,62 \mathrm{~km}^{2}$. El patrón de lluvias es el característico del Valle Central; la época seca va de enero a abril y la lluviosa de mayo a noviembre; pertenece a la zona de vida de Bosque Premontano Húmedo (Rodríguez \& Silva, 2015).

Se diseñó una encuesta conformada por 21 preguntas sobre prácticas de uso del agua, técnicas para disminuir el consumo de agua potable y principales fuentes de contaminación de ésta. Esta encuesta incluye tanto preguntas abiertas como cerradas. El instrumento se aplicó al 100\% (92) de las viviendas ubicadas en las urbanizaciones Orlich y El Laguito, aledañas a El Laguito, las cuales vierten sus aguas residuales directamente al embalse o a la quebrada El Estero.

El agua residual proveniente de las urbanizaciones ingresa a El Laguito por tres puntos: por una alcantarilla ubicada al lado oeste, por medio del caño ubicado en la

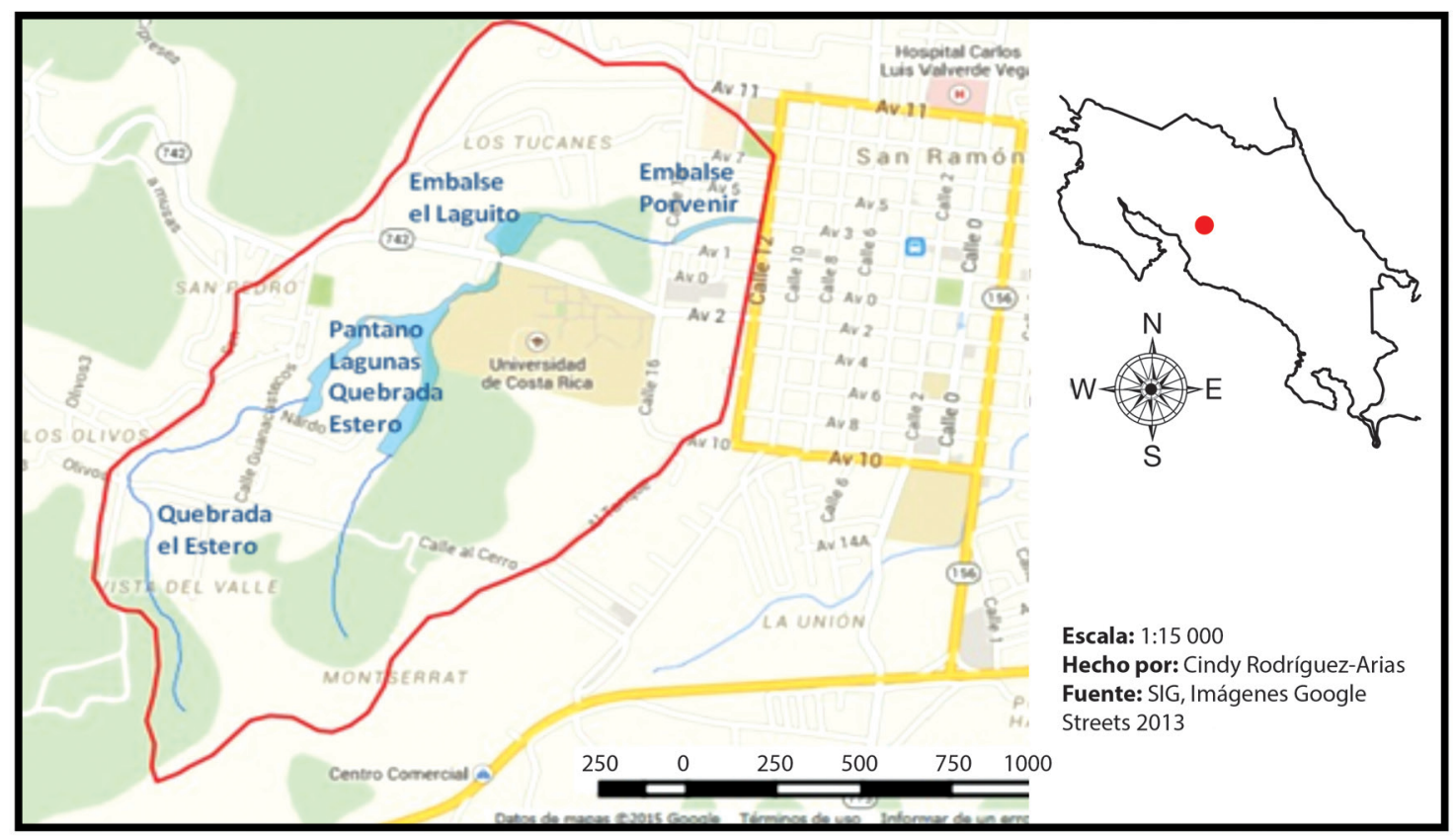

Fig. 1. Ubicación de El Laguito dentro de la microcuenca alta de la quebrada El Estero. 
carretera principal o por la quebrada Estero. Para validar la información obtenida de las encuestas, se analizó el agua que ingresa por la alcantarilla y el caño. El agua que ingresa por la quebrada no se analizó debido a que ya existen estudios sobre ésta. Se tomaron tres muestras en la alcantarilla y el caño, una en época seca (abril 2015) y dos en época lluviosa (setiembre 2015 y julio 2016). Las mismas fueron tomadas en horas de la mañana, sin lluvia.

Se evaluaron parámetros físico-químicos como la demanda biológica de oxígeno (DBO), la demanda química de oxígeno (DQO), los sólidos suspendidos totales, los sólidos sedimentables, las grasas y aceites, el $\mathrm{pH}$ y las sustancias activas al azul de metileno (SAAM). Para la recolección de cada muestra se utilizó el muestreo simple en envases de 2L. Antes de tomar la muestra, se enjuagó la botella con el agua a muestrear. Luego fue etiquetada con la fecha, hora, identificación del lugar y el punto de muestreo. Fueron almacenadas y transportadas al laboratorio de Agroanálisis de Costa Rica donde se analizaron.

\section{RESULTADOS}

Prácticas en el uso de agua: Se determinó que existen inadecuadas prácticas en el uso del agua lo cual provoca que la cantidad que ingresa al El Laguito sea alta. El $56 \%$ (51) de las personas encuestadas exceden el tiempo recomendado de baño sugerido por la OMS, el cual es de 5 a 10 minutos (Fig. 2).

Además, el 19\% (17) de los encuestados no acostumbran cerrar la llave de agua mientras se cepillan los dientes, cuando se enjabonan el cuerpo, mientras se bañan o se lavan las manos (Fig. 3).

Por otro lado, el 63\% (58) de los encuestados no utilizan ningún dispositivo para reducir el consumo de agua del servicio sanitario. De las 47 familias que utilizan manguera para lavar corredores y patios, el 34\% (16) no utiliza pistola, lo cual provoca un mayor desperdicio de agua. Finalmente, el 24\% (22) utilizan la lavadora una vez a la semana, 38\% (35) dos veces a la semana y 38\% (35) tres o más veces.

Técnicas para disminuir el consumo de agua potable: No existe en la población una costumbre de recoger el agua de la ducha, lavatorios o lavadoras para regar. Únicamente en el 15\% (14) de las viviendas se recoge algún tipo de agua para su posterior reutilización (Fig. 4).

Solamente en el 12\% (11) de las viviendas utilizan algún sistema de recolección de aguas pluviales (Fig. 5).

Fuentes de contaminación: Las principales fuentes de contaminación son el uso de detergentes no biodegradables y el vertido de aguas grises al caño o la quebrada. En el 96\% (88) de las viviendas utilizan jabón en polvo no biodegradable para el lavado de la ropa. El $80 \%$ (74) de las viviendas vierten las aguas grises directamente al caño o a la quebrada (Fig. 6).

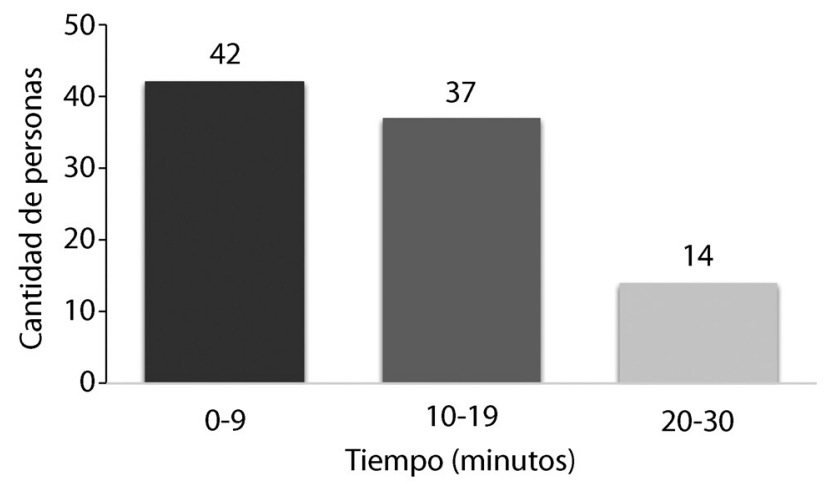

Fig. 2. Tiempo que dura cada persona bañándose.

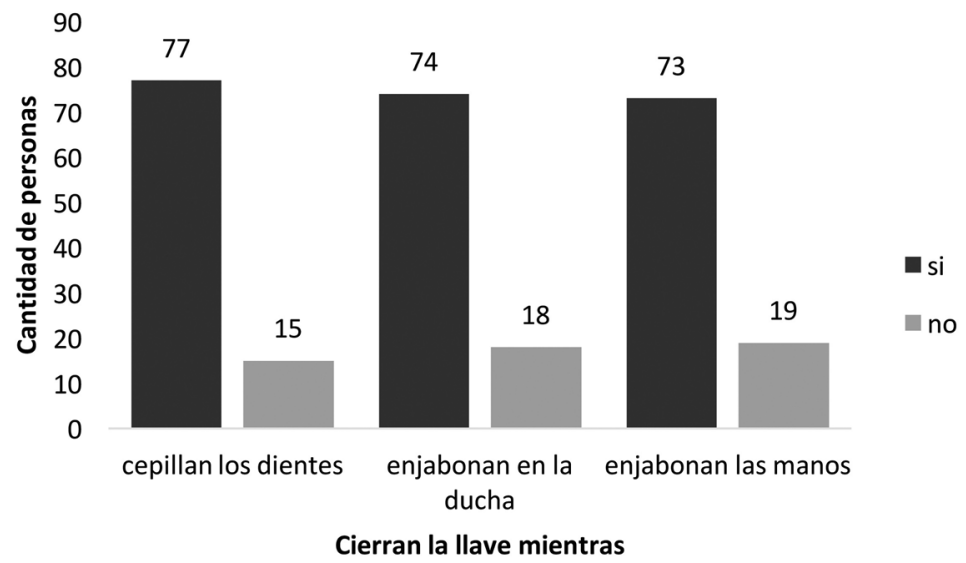

Fig. 3. Práctica de uso de agua mientras se lavan los dientes, bañan o lavan las manos. 


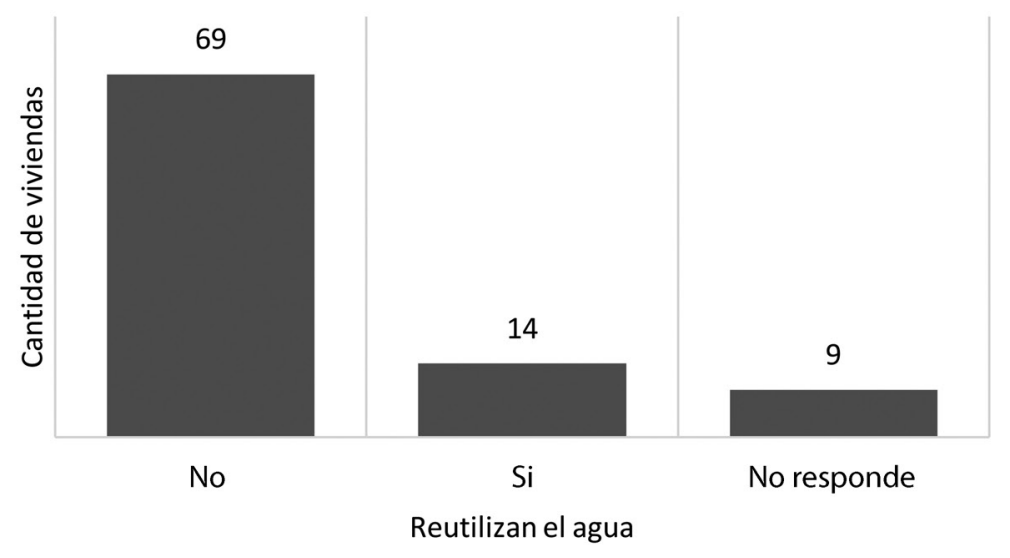

Fig. 4. Cantidad de personas que reutilizan el agua.

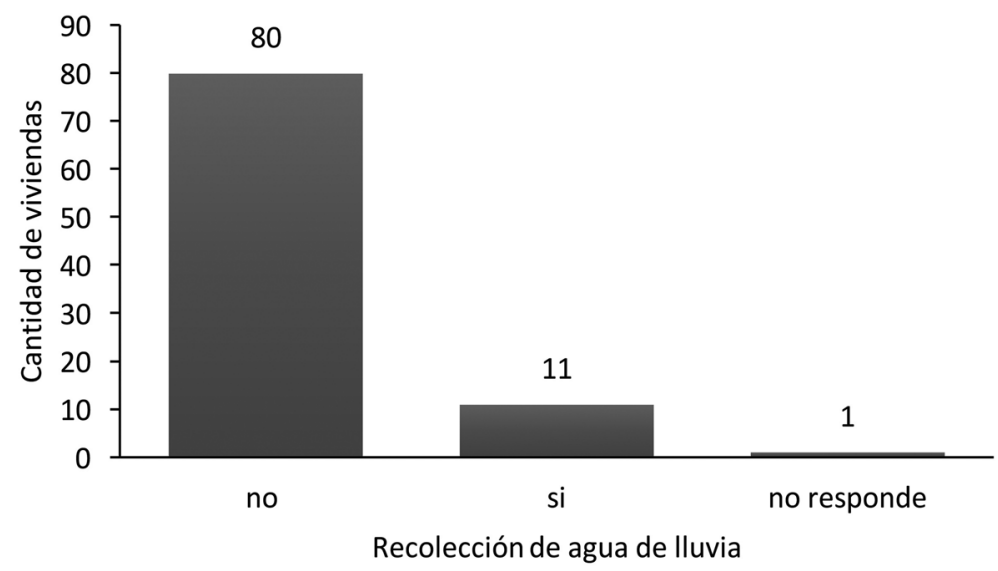

Fig. 5. Cantidad de personas que utilizan un sistema de recolección de aguas pluviales.

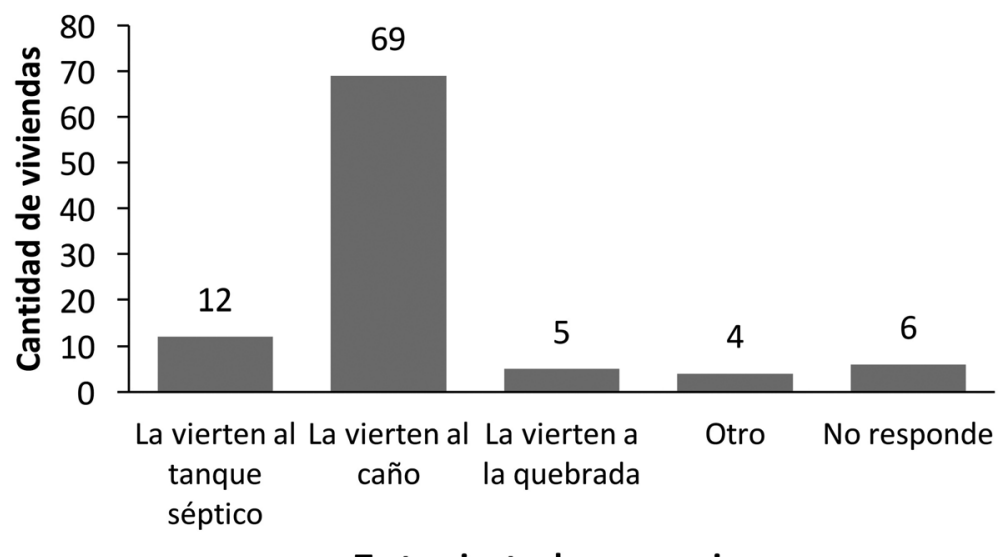

Fig. 6. Tratamiento de aguas grises. 
CUADRO 1

Comparación de los valores promedio obtenidos en la alcantarilla y el caño que ingresan a El Laguito para los parámetros físico-químicos con lo establecido por el Reglamento de vertido y reúso de aguas residuales. San Ramón, Alajuela, 2015 -2016

\begin{tabular}{lccc}
\multicolumn{1}{c}{ Parámetro } & \multicolumn{2}{c}{ Valor obtenido } & Valor reglamentado \\
DBO $(\mathrm{mg} / \mathrm{L})$ & Alcantarilla & Caño & 50 \\
DQO $(\mathrm{mg} / \mathrm{L})$ & 293 & 163 & 150 \\
Sólidos suspendidos $(\mathrm{mg} / \mathrm{L})$ & 603 & 291 & 50 \\
Sólidos sedimentables $(\mathrm{ml} / \mathrm{L})$ & 64 & 26 & 1 \\
Grasas y aceites $(\mathrm{mg} / \mathrm{L})$ & 4 & 1 & 30 \\
$\mathrm{pH}$ & 50 & 32 & 9 \\
SAAM $(\mathrm{mg} / \mathrm{L})$ & 7 & 7 & 2 \\
\hline
\end{tabular}

Las aguas negras son tratadas con tanque séptico en el $100 \%$ de los casos. De éstos el $68 \%$ tienen drenaje y el $90 \%$ se encuentran en buenas condiciones

Parámetros fisicoquímicos del agua procedente de las viviendas: Según el Reglamento de Vertido y Reúso de Aguas Residuales No. 33601 (Ministerio de Ambiente y Energía y Ministerio de Salud, 2007), todos los parámetros analizados exceden los valores permitidos (Cuadro 1).

\section{DISCUSIÓN}

En Costa Rica se calcula que una persona que vive en una ciudad utiliza en promedio $250 \mathrm{~L}$ de agua por persona por día, de los cuales aproximadamente $180 \mathrm{~L}$ se convierten en aguas residuales grises. Además, las prácticas sanitarias promovidas actualmente se basan en la construcción de tanques sépticos para el depósito de aguas grises y negras, en el vertido directo en los cauces de los ríos empleando letrinas o alcantarillados sanitarios o en el envío por sistemas de tuberías a plantas de tratamiento. Esta última opción es la que es menos utilizada debido a sus altos costos (Moreno, 2009), lo cual ocasiona que la cantidad y calidad del agua que se vierte a los cuerpos de agua sea alta y contaminada.

En el caso descrito en este artículo, se observa que el comportamiento de los encuestados no es diferente al comportamiento nacional. El $56 \%$ de las personas entrevistadas duran más de 10 minutos bañándose, por lo que consumen solo en esta actividad más de $200 \mathrm{~L}$ de agua por día (Cadena SER, 2016). El 63\% no utilizan ningún dispositivo para reducir el consumo de agua del servicio sanitario, lo cual implica el uso aproximado de $50 \mathrm{~L}$ de agua potable por persona por día. El 38\% de las familias usan la lavadora tres o más veces por semana, lo que consume en promedio $285 \mathrm{~L}$ de agua por lavada (Superintendencia Servicios Sanitarios, 2007). Todo esto ocasiona que, a El Laguito, sólo por la alcantarilla proveniente de estas urbanizaciones, estén ingresando aproximadamente $26 \mathrm{~m}^{3}$ de aguas residuales por día con una contaminación superior a lo permitido por el reglamento de vertido y reúso de aguas residuales. El DBO es seis veces mayor a lo establecido, el DQO cuatro veces, los SST uno con tres décimas, las grasas y aceites uno con siete décimas y las SAAM 12,5 veces. Muy similar es la situación que se presenta en el agua que ingresa por el caño, en la cual el DBO es tres veces mayor a los permitido, el $\mathrm{DQO}$ dos veces, las grasas y aceites uno con una décima y las SAAM 23 veces.

Otro punto de ingreso de agua a El Laguito es la quebrada Estero, la cual como demostraron Bolaños, Montero, Rodríguez y Sánchez (2015) también está contaminada. El punto más cercano a El Laguito, muestreado por ellos, presenta en promedio un DBO de $317 \mathrm{mg} / \mathrm{L}$ y un DQO de $765 \mathrm{mg} / \mathrm{L}$. De acuerdo a Mejía, Rosales, Rojas y Molina (2006) y a la Comisión Nacional de Agua, 2017, la presencia en el agua de un DBO mayor a $120 \mathrm{mg} / \mathrm{L}$ y un DQO mayor a $200 \mathrm{mg} / \mathrm{L}$ las clasifica como fuertemente contaminadas y se consideran como aguas superficiales con un fuerte impacto de descargas de aguas residuales crudas municipales y no municipales. Situación que se comprueba en los tres puntos de ingreso de agua a El Laguito.

También Rodríguez y Silva (2015) midieron el grado de contaminación en la quebrada Estero y en El Laguito utilizando el Índice del Sistema Holandés de Calidad del Agua (IHCA-CR), evidenciando contaminación en ambos lugares, tanto en verano como en invierno. En época seca la quebrada presentó contaminación de moderada a muy severa y El Laguito contaminación moderada. 
En época lluviosa la quebrada presentó contaminación de moderada a incipiente y El Laguito incipiente. La calidad del agua superficial difiere según la estación del año, mostrando mayores índices de contaminación en la época seca, lo cual es causado por una mayor concentración de los contaminantes en el agua al disminuir el caudal (Calvo y Mora, citados por Rodríguez y Silva, 2015). La quebrada Estero mantiene el mínimo caudal en los meses de febrero y marzo, periodo en el cual el mayor aporte lo constituyen las aguas residuales provenientes de residencias, comercios y servicios existentes en la microcuenca, mientras que en el periodo lluvioso hay un mayor caudal como producto de las precipitaciones pluviales (Quesada, 2001).

A pesar del ingreso de contaminantes a El Laguito por el caño, la quebrada y la alcantarilla, éste presenta contaminación moderada en verano e incipiente en invierno. Esto se debe al aumento del caudal y consecuente aumento de la aireación y al paso del agua atreves de la vegetación que disminuye la carga de nutrientes (Rodríguez y Silva, 2015), ya que éstas tienen una alta capacidad para remover grandes concentraciones de contaminantes (Nahlik \& Mitsch, 2006).

Podría entonces decirse que la situación de El Laguito, en cuanto al nivel de contaminación, es aceptable, sin embargo, tomando en cuenta otros parámetros como el porcentaje de oxígeno disuelto (POD) y las coliformes, la situación cambia. Según Pérez-Castillo y Rodríguez (como se citó en Rodríguez y Silva, 2015), el POD es muy importante en los ecosistemas acuáticos porque define en gran parte la biodiversidad y la supervivencia de la comunidad biótica. La mayoría de los peces toleran una concentración baja de oxígeno disuelto por cierto periodo de tiempo y reducciones por debajo del porcentaje de saturación generan efectos negativos sobre la biodiversidad, el crecimiento, la reproducción y la actividad de ellos. En el caso de El Laguito, en ambas épocas, los valores promedio de POD presentaron valores inferiores al $60 \%$, porcentaje mínimo recomendado para aguas superficiales, lo cual limita la presencia de biodiversidad en éste. Una de las causas de que la concentración de oxígeno baje es la presencia de aguas residuales.

Además, el embalse ha perdido el volumen de almacenamiento y el espejo de agua, debido al arrastre, a la deposición de sedimentos y a la eutrofización ocasionada por las descargas de aguas residuales domésticas, lo cual disminuye la posibilidad para atenuar caudales y provoca desbordamientos. Esto se agrava durante los eventos extremos de precipitación por la influencia de factores naturales como El Niño (ENOS), La Niña, movimientos latitudinales del Centro de Convergencia
Intertropical, tormentas tropicales y huracanes, la influencia del Atlántico y los empujes fríos (Rodríguez, 2017), aumentando el riesgo de inundación de la ciudad de San Ramón.

Del estudio se concluye que la población cercana a EI Laguito presenta malas prácticas en el uso, tratamiento y disposición del agua, lo que provoca que a El Laguito y a la quebrada Estero estén llegando grandes cantidades de agua contaminada. Específicamente para remediar el problema de la contaminación del agua que ingresa por la alcantarilla, se diseñó y construyó una biojardinera para tratar las aguas antes de que éstas ingresen al embalse. Las biojardineras son sistemas de fitodepuración de aguas residuales (Delgadillo, Camacho, Pérez y Andrade, 2010), las cuales consisten en el desarrollo de plantas enraizadas sobre un lecho de piedra impermeabilizado cuya meta es la reducción de contaminantes específicos a un nivel aceptable. La depuración del agua ocurre por la interacción entre los elementos componentes de la biojardinera y de los fenómenos físicos, químicos y biológicos que se dan dentro de ésta con la intervención del sol como fuente de energía (Arias y Brix, 2003). Permiten disminuir la contaminación del agua entre un $70 \%$ y $90 \%$. La medición de la eficiencia de las biojardineras construidas está en proceso.

Pero, aunque esta biojardinera ayude en la disminución de la contaminación, es una medida paliativa, que solo trata un punto de ingreso de aguas contaminadas, por lo que, para mejorar la situación y atacar la causa es necesario plantear una estrategia que involucre a todas las instituciones que tienen injerencia en el tema, como son la Sede de Occidente de la UCR, el A y A, la Municipalidad de San Ramón, Ministerio de Salud y Asociaciones de Desarrollo. Esto fue realizado por Rodríguez (2017) quién diseñó un plan de rehabilitación y conservación de los humedales en la microcuenca alta de la quebrada Estero, la cual incluye a El Laguito. Este plan propone estrategias tendientes a la rehabilitación ecológica de los humedales que permitan restablecer la provisión de servicios ecológicos para mitigar inundaciones, crear hábitats para la vida silvestre, la recreación, educación ambiental e investigación científica, poniendo en marcha seis programas: régimen hidrológico, hidroperiodo y topografía; calidad del agua; suelo y sedimentos; especies exóticas, invasoras y depredadores domésticos; flora y fauna nativas y participación social.

\section{AGRADECIMIENTOS}

Las autoras expresan su agradecimiento a la Coordinación de Investigación de la Sede de Occidente 
de la UCR por el apoyo financiero y logístico. A Edwin Barrantes Barrantes, profesor encargado del TCU 647 Rescate de la microcuenca quebrada El Estero, por el apoyo en la aplicación de las encuestas.

\section{REFERENCIAS}

Arias, C. y Brix, H. (2003). Humedales artificiales para el tratamiento de aguas residuales. Ciencia e Ingeniería Neogranadina, 13, 17-24. Recuperado de: http://www. redalyc.org/articulo.oa?id $=91101302$

Bolaños, Montero, Rodríguez y Sánchez (2015). Calidad de aguas superficiales: estudio de la quebrada Estero, ubicada en el cantón de San Ramón, Costa Rica. Revista Pensamiento Actual, 15(25), 61-76

Cadena SER (2016). ¿Cuánta agua utilizamos durante la ducha? .Recuperado de: http://cadenaser.com/ser/2014/01/20/ sociedad/1390187610_850215.html

Chulluncuy, N. (2011). Tratamiento de agua para consumo humano. Ingeniería Industrial, 29, 153-170. Recuperado de: https://revistas.ulima.edu.pe/index.php/ Ingenieria_industrial/article/download/.../208

Comisión Nacional del Agua (2017). Monitoreo de calidad del agua. Recuperado de: http://files.conagua.gob.mx/ transparencia/CalidaddelAgua.pdf

Delgadillo, O., Camacho, A., Pérez, L, \& Andrade, M. (2010). Depuración de aguas residuales por medio de humedales artificiales. Recuperado de: http://www.infoandina.org/ sites/default/files/publication/files/depuracion_de_ aguas_residuales_por_medio_de_humedales_artificiales.pdf

Estrada, R., López, M. G., Vásquez, R., Sánchez, D., \& Ruvalcaba, J. C. (2016). Conocimiento y percepción respecto al impacto de vivir cerca de canales de aguas residuales. Journal of Negative and No Positive Results, 1(4), 142-148.

Mejía, E., Rosales, F., Rojas, J., \& Molina, M. (2006). Atlas de la cuenca Lerma-Chapala construyendo una vision conjunta. Instituto Nacional de Ecología, México. Recuperado de http://www2.inecc.gob.mx/emapas/download/lch_ calidad_del_agua.pdf
MINAET, SENARA, \& AyA. (2012). "Agenda del Agua" Costa Rica 2013 - 2030. Recuperado de:http://www.gwp.org/globalassets/global/gwp-cam_files/documento_de_posicionamiento_agenda_del_agua_nov_20121.pdf

Ministerio de ambiente y energía y Ministerio de Salud (19 de marzo de 2007). Reglamento de Vertido y Reúso de Aguas Residuales. Recuperado de http://www.pgrweb.go.cr/ scij/Busqueda/Normativa/Normas/nrm_texto_completo.aspx?param1=NRTC\&nValor1=1\&nValor2=59524\&nValor3=83250\&strTipM=TC

Mora, C. (2007). Nueva propuesta de diseño para el embalse de regulación y uso múltiple "El Laguito", con el fin de prevenir las inundaciones en la ciudad de San Ramón. (Tesis de licenciatura) Universidad de Costa Rica, San José, Costa Rica.

Moreno, L. (2009). Valoración económica del uso de tecnologías de saneamiento ecológico para aguas residuales domiciliares. Revista Iberoamericana de Economía Ecológica, 13, 1-13.

Nahlik, A. M., \& Mitsch, W. J. (2006). Tropical treatment wetlands dominated by freefloating macrophytes for water quality improvement in Costa Rica. Ecological Engineering, 28(3), 246-257.

Quesada, M. (2001). Evaluación socioambiental de la microcuenca quebrada Estero, San Ramon, Costa Rica. Coordinación de Investigación, Sede de Occidente de la Universidad de Costa Rica, Costa Rica.

Rodríguez, C. (2017). Plan de rehabilitación ecológica, manejo y conservación de los humedales de la quebrada El Estero, San Ramón, Alajuela (Tesis doctoral). DOCINADE, Costa Rica.

Rodríguez, C., \& Silva, M. (2015). Calidad del agua en la microcuenca alta de la quebrada Estero en San Ramón de Alajuela, Costa Rica. Revista Pensamiento Actual, 15(25), 85-97.

Superintendencia de servicios sanitarios - SISS. (2007). Manual para el consumo responsable de agua potable. Recuperado de: http://www.siss.gob.cl/577/articles-9103_recurso_1.pdf 